\title{
Kilo-Tesla axial magnetic field generation with high intensity spin and orbital angular momentum beams
}

\author{
Andrew Longman (1)* \\ Lawrence Livermore National Laboratory, Livermore, California 94551, USA \\ Robert Fedosejevs (1) \\ Department of Electrical and Computer Engineering, University of Alberta, Edmonton, Alberta, Canada T6G1R1
}

(Received 16 September 2021; accepted 22 November 2021; published 14 December 2021)

\begin{abstract}
Absorption of angular momentum from a high intensity laser pulse can lead to the generation of strong axial magnetic fields in plasma. The effect, known as the inverse Faraday effect, can generate kilo-Tesla strength, multipicosecond, axial magnetic fields extending over hundreds of microns in underdense plasma. In this paper we explore the effect with ultrahigh intensity circularly polarized Gaussian beams and linearly polarized orbital angular momentum beams comparing analytic expressions with three-dimensional particle-in-cell simulations. We develop a model for the transverse magnetic field profiles, introduce a model for the temporal decay, and show that while the magnetic field strength is independent of plasma density, it has a strong dependence on the laser beam waist.
\end{abstract}

DOI: 10.1103/PhysRevResearch.3.043180

\section{INTRODUCTION}

The inverse Faraday effect (IFE) describes the generation of axial magnetic fields when angular momentum is transferred from a laser pulse to plasma. If the pulse is circularly polarized $(\mathrm{CP})$ and of relativistic intensity, the generated magnetic field strength can be on the order of hundreds of Teslas, can last several picoseconds, and can extend over millimeter scales [1]. The IFE has been studied extensively with $\mathrm{CP}$ beams both theoretically [2-8] and experimentally $[1,9,10]$, yet the effect is not exclusive to CP beams but also applicable to lasers carrying orbital angular momentum (OAM) [11-13]. Currently, we know of only one analytic model describing OAM driven magnetic fields and its transverse profile [11], but it has not been verified numerically or experimentally. Furthermore, little is known about the axial extent of the magnetic fields or their lifetimes for both CP and OAM drivers.

There are several advantages of using OAM beams in high powered lasers as opposed to $\mathrm{CP}$ beams: unlike CP beams that carry a maximum spin angular momentum of $\sigma_{z} \hbar$ per photon, where $\sigma_{z}=\{ \pm 1\}$ is the spin number $\left[\sigma_{z}=0\right.$ for linearly polarized (LP) beams], OAM beams carry an angular momentum of $\left(\sigma_{z}+\ell\right) \hbar$ per photon, where $\ell$ is an azimuthal mode number that can take any integer value [14]. OAM has also been shown to couple more efficiently to free electrons due to an optimal overlap between the laser intensity profile

\footnotetext{
*longman1@1ln1.gov

Published by the American Physical Society under the terms of the Creative Commons Attribution 4.0 International license. Further distribution of this work must maintain attribution to the author(s) and the published article's title, journal citation, and DOI.
}

and the angular momentum density, yielding greater coupling at larger radial distances [15]. Additionally, high intensity OAM beams have recently been produced with high power lasers using off-axis spiral phase mirrors mitigating nonlinear effects like temporal distortions and the $B$ integral present with transmissive optics such as quarter wave plates [16].

Recent works have looked at simulating IFE driven magnetic fields from OAM beams in various configurations: OAM beams with radial and azimuthal polarizations [12], amplification of seeded magnetic fields [17], spatiotemporal light springs [18], and, most recently, linearly polarized OAM beams [13]. In these studies the laser intensities were of moderate intensity $\left(I_{0} \approx 10^{18} \mathrm{~W} \mathrm{~cm}^{-2}\right)$, verifying the existence of weaker magnetic fields $(\approx 10 \mathrm{~T})$, with little modeling of the spatial or temporal properties of the magnetic fields.

In this work, we explore the spatial and temporal scaling of IFE magnetic fields driven by ultrahigh intensity $\left(I_{0} \approx\right.$ $10^{20} \mathrm{~W} \mathrm{~cm}^{-2}$ ) CP Gaussian and LP OAM beams. We develop analytic models for both the spatial and temporal scales of the magnetic field and verify them with full three-dimensional (3D) relativistic particle-in-cell (PIC) simulations. We demonstrate strong coupling of the laser angular momentum to the plasma through ponderomotive forces, resulting in axial magnetic fields in excess of $1 \mathrm{kT}$, more than $200 \mu \mathrm{m}$ in length, and lasting several picoseconds, in good agreement with the predictions of our analytic models and previous experiments using CP Gaussian beams.

\section{COUPLING OF HIGH INTENSITY ANGULAR MOMENTUM TO PLASMA}

A circularly polarized beam is classically defined by its polarization vector rotating about the laser propagation axis $k_{z}$ in either a clockwise or counterclockwise direction. Similarly, a 
beam carrying a well-defined OAM is typically characterized by a helical wave front also orientated about the $k_{z}$ vector with the helix rotating in either a clockwise or counterclockwise direction [14]. Unlike a Gaussian beam, paraxial focusing of an OAM beam produces a complicated field structure described by modified-Bessel or hypergeometric functions but can be modeled using a suitably chosen basis set such as the Laguerre-Gaussian (LG) modes [19]. The electric field magnitude of a general OAM laser pulse can then be approximately given by $E=E_{0} \psi_{\ell} g(t) \exp \left[i\left(\omega_{0} t-k_{z} z\right)\right]$, where $E_{0}$ is the peak electric field strength of the fundamental Gaussian mode $(\ell=0), g(t)$ is the temporal envelope, and $\omega_{0}$ is the laser frequency. $\psi_{\ell}$ are the azimuthal LG modes (zero-radial mode) and can be given in the following compact form $[14,20]$ :

$$
\psi_{\ell}=\frac{1}{\sqrt{|\ell| !}}\left(\frac{r \sqrt{2}}{w_{0}}\right)^{|\ell|}\left(\frac{z_{0}}{Z}\right)^{|\ell|+1} \exp \left(-\frac{k r^{2}}{2 Z}\right) e^{i \ell \phi},
$$

where $w_{0}$ is the beam waist in the focal plane, $z_{0}=k w_{0}^{2} / 2$ is the Rayleigh range, and $Z=z_{0}+i z$ is the complex beam parameter. Taking the modulus squared of the field amplitude given in Eq. (1) for $|\ell| \geqslant 1$, we find the so-called donut mode intensity profiles that are symmetric about the $k_{z}$ axis; the helical phase is lost in the modulus, resulting in no azimuthal structure in the intensity profile [14]. Increasing the azimuthal mode integer $|\ell|$ increases the OAM in the beam and increases the donut radius, and by conservation of energy, the peak intensity around the donut decreases for a fixed laser energy and focusing geometry [21]. As the peak intensity varies for different values of $|\ell|$ in a nontrivial way, we opt to use the beam power instead as it is constant for all $|\ell|$ modes.

The ponderomotive force acting on an electron associated with a high intensity focal spot can be given by $\boldsymbol{F}_{p}=$ $-m_{e} c^{2} \nabla \sqrt{1+|\boldsymbol{a}|^{2}}$, where $\boldsymbol{a}=e \boldsymbol{E} / m_{e} c \omega_{0}$ is the normalized vector potential, $e$ and $m_{e}$ are the electron charge and mass, respectively, and $c$ is the speed of light. In the standard case of a high intensity Gaussian beam finite energy maybe transferred from the laser to plasma electrons through ponderomotive scattering [22]. The same is true for higher order LG modes [23], with some possibility for electron trapping and additional energy transfer within the donut mode leading to a possible increased absorption rate [24,25]. However, ponderomotive scattering to first order is an intensity driven phenomenon, and no OAM is transferred to the plasma as the LG mode intensity has no azimuthal component.

Recent work has analyzed the motion of free electrons in a LG beam in more detail, expanding to first and second order perturbations $[13,15]$. It was shown that angular momentum can be transferred to free electrons in LP LG beams when considering second order terms in the equations of motion; the electron gains and loses angular momentum, but averaging over one laser cycle yields zero net angular momentum transfer. An asymmetry in the laser field such as the ponderomotive force or collisions are therefore needed to transfer a net OAM to the electrons.

\section{THE INVERSE FARADAY EFFECT}

To model the IFE, we start with the angular momentum conservation model used by Haines [3]. In this model, cou- pling of the angular momentum density in the plasma with that of a driving laser is performed through an arbitrary absorption parameter, yielding the conservation equation

$$
\begin{aligned}
n_{e} m_{e} r \frac{d v_{e \theta}}{d t}= & -n_{e} e r E_{\theta}-n_{e} \operatorname{er}\left(v_{e z} B_{r}-v_{e r} B_{z}\right) \\
& +\alpha_{\mathrm{abs}} M_{z} c-n_{e} m_{e} v_{e i} r v_{e \theta} .
\end{aligned}
$$

Here, $n_{e}$ is the electron density, $\alpha_{\mathrm{abs}}$ is the laser absorption fraction per unit length, $M_{z}$ is the laser angular momentum density, $v_{e r}$ and $v_{e \theta}$ are the radial and azimuthal electron velocities, respectively, and $\nu_{e i}$ is the electron-ion collision frequency. $E_{\theta}, B_{r}$, and $B_{z}$ are the plasma azimuthal electric, radial magnetic, and axial magnetic fields, respectively. Assuming the electrons are in steady state, ignoring the inertial and collisional terms, and using Faraday's law, we obtain the time rate of change of the axial magnetic field approximately as [3]

$$
\frac{\partial B_{z}}{\partial t} \approx \frac{c}{r e} \frac{\partial}{\partial r} \frac{\alpha_{\mathrm{abs}} M_{z}}{n_{e}} .
$$

For an OAM mode that is either CP or LP, the angular momentum density can be given by $[11,14]$

$$
M_{z}=\frac{I_{0}\left|\psi_{\ell}\right|^{2} g(t)^{2}}{\omega_{0} c}\left[\ell+\sigma_{z}\left(|\ell|-\frac{2 r^{2}}{w_{0}^{2}}\right)\right] .
$$

Radial differentiation of Eq. (4) is straightforward, and if we assume a Gaussian temporal function, then the integration of Eq. (3) is trivial given $\int g(t)^{2} d t \approx 3 \tau / 4$, where $\tau$ is the temporal full width at half maximum (FWHM). By introducing the pulse temporal function $g(t)$, we are limiting the IFE to be driven only when the laser field is present. Although the magnetic field does not instantaneously disappear after the laser has passed, its evolution is no longer governed by the IFE, but rather the plasma dynamics, which will be discussed later.

Substituting Eq. (1) into Eqs. (3) and (4), assuming the laser absorption rate and electron density are constant in space and time (no ponderomotive channeling), assuming a Gaussian temporal pulse shape, and substituting the laser power $P=\pi I_{0} w_{0}^{2} / 2$, we obtain the following expression for the axial magnetic field:

$$
\begin{aligned}
B_{z}= & \frac{3 \alpha_{\mathrm{abs}} P \tau}{\pi e n_{e} \omega_{0} w_{0}^{4}}\left|\psi_{\ell}\right|^{2} \\
& \times\left[\ell\left(\frac{|\ell| w_{0}^{2}}{r^{2}}-2\right)+\sigma_{z}\left(\frac{|\ell|^{2} w_{0}^{2}}{r^{2}}-4|\ell|+\frac{4 r^{2}}{w_{0}^{2}}-2\right)\right] .
\end{aligned}
$$

The direct proportionality on $\ell$ and $\sigma_{z}$ in Eq. (5) indicates that the axial magnetic field direction can be controlled by changing the sign of OAM helicity or the CP handedness. The helicity of a LP OAM mode, the magnetic field direction, and the corresponding electron motion are illustrated in Fig. 1.

Several transverse magnetic field profiles are plotted in Fig. 2 with laser plasma parameters $n_{e}=0.03 n_{c}, \lambda=1 \mu \mathrm{m}$, $w_{0}=6 \mu \mathrm{m}, \alpha_{\mathrm{abs}}=0.435 \mathrm{~mm}^{-1}, \tau=100 \mathrm{fs}$, and $P=$ $65 \mathrm{TW}$, where $\lambda$ is the laser wavelength and $n_{c}$ is the critical plasma density. We note that the $\left(\left|\sigma_{z}\right|=1,|\ell|=0\right)$ and $\left(\sigma_{z}=\right.$ $0,|\ell|=1)$ modes produce the same magnetic field profiles 


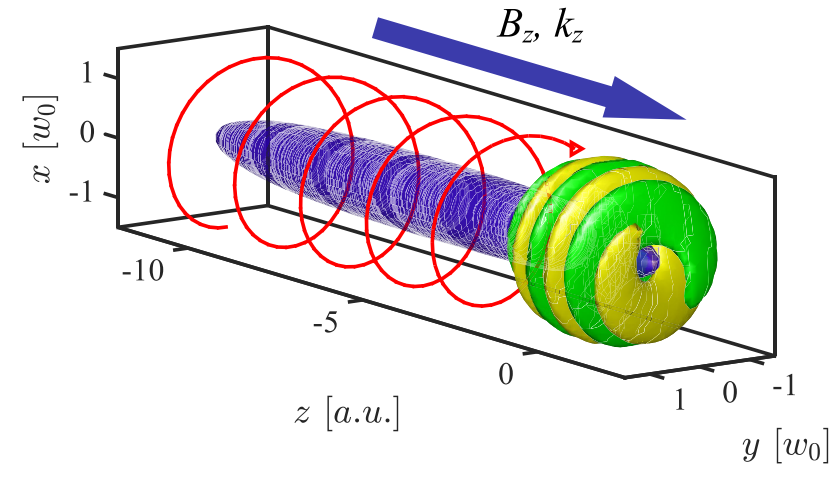

FIG. 1. Illustration of an IFE driven $B$ field with a LP $\ell=1$ OAM mode and the corresponding electron helical trajectory in red, with the arrowhead showing the velocity direction. The green and yellow isosurfaces show the positive and negative electric fields of the laser, respectively, while the blue isosurface shows the positive magnetic field direction, as indicated by the arrow.

peaked on axis, while the higher order $\ell$ modes produce more complex coaxial structures that are zero on axis. Given the magnetic field peak on axis and the higher magnetic field strength, the $\left(\left|\sigma_{z}\right|=1,|\ell|=0\right)$ and $\left(\sigma_{z}=0,|\ell|=1\right)$ driven fields are probably more desirable for most applications. For the purpose of this work, we do not consider beams that are circularly polarized and have OAM, but we remark that a beam with $\left(\sigma_{z}= \pm 1, \ell= \pm 1\right)$ produces the strongest on-axis magnetic field with a peak field strength double that of the $\left(\left|\sigma_{z}\right|=1,|\ell|=0\right)$ case. Using these parameters and assuming the laser is entirely absorbed, the peak ratio of the energy per unit length contained in the magnetic field to the laser energy per unit length is roughly $0.2 \%$ for the $\mathrm{CP} \ell=0$ and LP $|\ell|=1$ cases, slightly increasing for higher order $\ell$ modes.

The dependence of $\alpha_{\text {abs }}$ on $\ell$ is not well understood for any absorption mechanism. At laser intensities of $I \lambda^{2} \geqslant$ $10^{19} \mathrm{~W} \mathrm{~cm}^{-2} \mu \mathrm{m}^{2}$ and pulse durations greater than $100 \mathrm{fs}$, we can estimate the pump depletion length in underdense

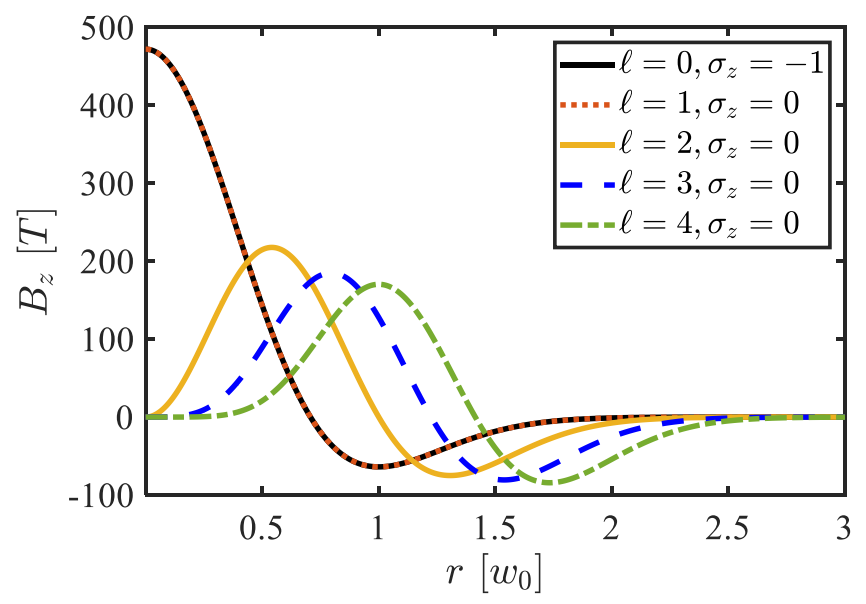

FIG. 2. Transverse IFE driven magnetic field profiles driven from a CP Gaussian beam and various LP OAM beams given by Eq. (5) and the legend. The following laser plasma parameters were used: $n_{e}=0.03 n_{c}, \lambda=1 \mu \mathrm{m}, P=65 \mathrm{TW}, w_{0}=6 \mu \mathrm{m}, \alpha_{\mathrm{abs}}=$ $0.435 \mathrm{~mm}^{-1}$, and $\tau=100 \mathrm{fs}$. plasma $\left(n_{e}>0.01 n_{c}\right)$ as $L_{p d}=n_{c} c \tau / n_{e}$, where $\tau$ is the temporal FWHM [26,27]. Rearranging, we obtain the absorption rate as

$$
\alpha_{\mathrm{abs}}=\frac{n_{e}}{2 n_{c} c \tau} .
$$

Given the absorption is independent of beam intensity and beam waist, we assume the model is approximately valid for low order OAM modes. If $\tau \gtrsim 100 \mathrm{fs}$ and $n_{e} \gtrsim 0.01 n_{c}$, we can use Eqs. (5) and (6) to estimate the peak axial magnetic field strength of the $\left(\left|\sigma_{z}\right|=1,|\ell|=0\right)$ and $\left(\sigma_{z}=0,|\ell|=1\right)$ modes,

$$
|B|_{\max } \approx 10 \frac{P[\mathrm{TW}] \lambda^{3}[\mu \mathrm{m}]}{w_{0}^{4}[\mu \mathrm{m}]} \mathrm{kT} .
$$

The fourth power dependence on the laser beam waist indicates a strong dependence on the beam $f$ number and relativistic self-focusing. Equation (7) should therefore be considered an underestimate as the beam will self-focus beyond the diffraction limit, giving rise to much stronger magnetic fields. Using Eq. (7), we can compare its prediction with the experimental results previously measured using CP Gaussian beams [1]. In that experiment, the Vulcan laser ( $40 \mathrm{TW}, 1 \mathrm{ps}, \lambda=1.054 \mu \mathrm{m}$ ) was focused into an underdense plasma, where it was observed to self-focus to a beam waist of $w_{0} \approx 5 \mu \mathrm{m}$. A peak magnetic field strength of $\approx 700 \mathrm{~T}$ was observed, in good agreement with our Eq. (7) estimate of $771 \mathrm{~T}$. The same experiment also measured the independence of the magnetic field strength from the plasma density, also in agreement with our model.

Circularly polarizing large diameter, high power laser beams is not trivial, requiring fragile and costly optics. However, with the new generation of PW class lasers coupled with off-axis spiral phase mirrors [16], axial magnetic fields on the order of tens of kilo-Teslas may be feasible using LP OAM modes. Ultimately, the field strength will depend on the absorption rate of the laser, which may diverge from our simple model when using ultrahigh intensity beams $\left(I_{0} \gtrsim\right.$ $10^{21} \mathrm{~W} \mathrm{~cm}^{-2}$, high $|\ell|$ beams, or ultrashort pulse durations ( $\tau<100 \mathrm{fs}$ ). Additionally, realistic diffraction of OAM beams when generated by spiral phase optics is more complex, leading to larger beam waists and lower intensities than one would expect using pure Gaussian beams [19,21]. While we do not include these modified beam waists and intensities here, it is simple to use the estimates from previous works to estimate the reduction in the magnetic field strength.

\section{SIMULATION RESULTS}

To simulate the IFE, we use the $3 \mathrm{D}$ relativistic PIC code $\mathrm{EPOCH}$ [28]. We assume a fully ionized helium plasma with a super-Gaussian longitudinal shape to mimic the electron density from a gas jet given by $n(z)=n_{e} \exp \{-[(z-$ $\left.350 \mu \mathrm{m}) / 300 \mu \mathrm{m}]^{10}\right\}$ with initial electron and ion temperatures of $1 \mathrm{keV}$ and $1 \mathrm{eV}$, respectively. Using this profile, the plasma ramps from vacuum to $n_{e}$ over roughly $100 \mu \mathrm{m}$. The laser is polarized along the $\hat{x}$ axis, has a Gaussian temporal pulse shape, and is focused on the plane $z=135 \mu \mathrm{m}$.

The laser pulse is tracked with a moving window $200 \mu \mathrm{m}$ long and $48 \times 48 \mu \mathrm{m}^{3}$ in the transverse directions with grid 
(a)

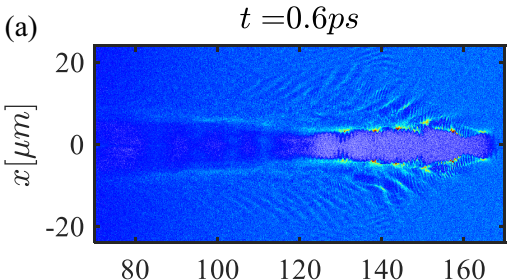

(b)
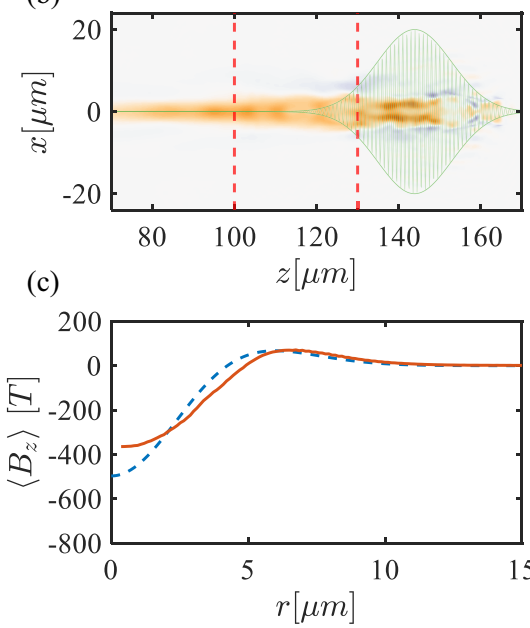

(d)

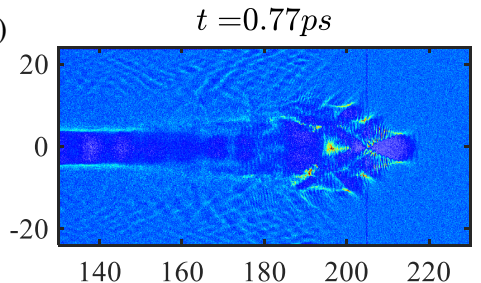

(e)

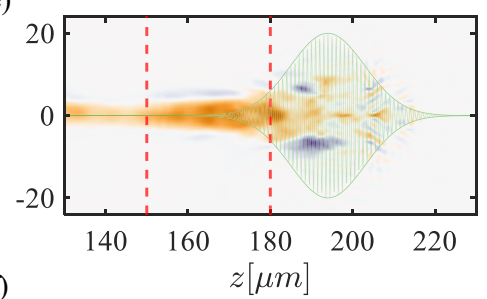

(g)

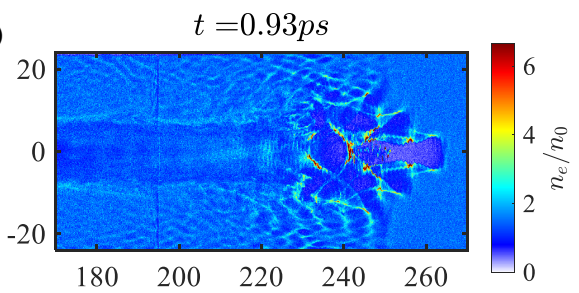

(h)

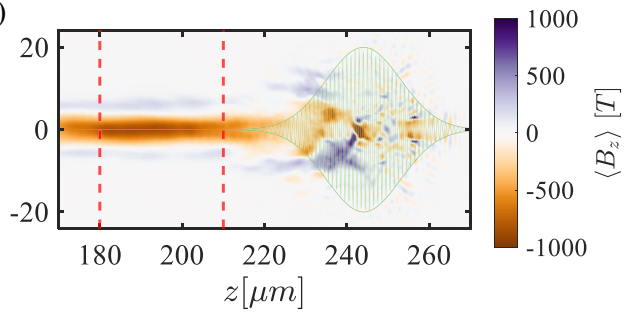

(i)
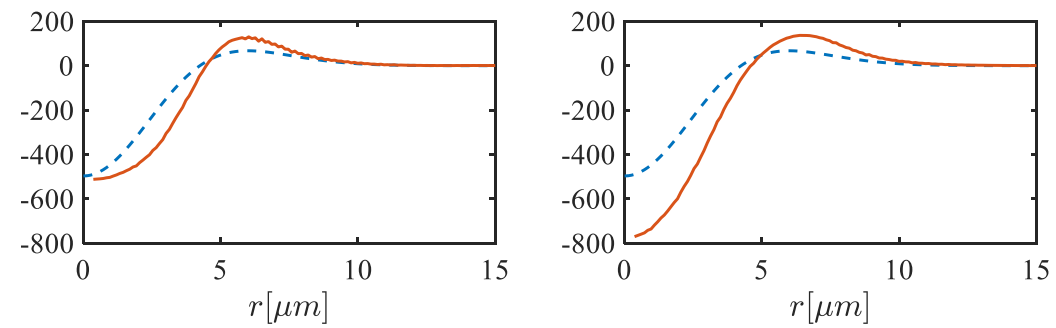

FIG. 3. Simulation results for CP Gaussian driven magnetic fields at (from left to right) $0.6,0.77$, and 0.93 ps. (a), (d), and (g) Longitudinal electron density slice maps normalized to the initial electron density. (b), (e), and (h) Longitudinal axial magnetic field slice maps averaged over $33 \mathrm{fs}$; the laser pulse is overlayed in green, and lineout regions are given by the red dashed lines. (c), (f), and (i) Radial axial magnetic field lineouts averaged azimuthally, temporally, and longitudinally between the red dashed lines in the corresponding panel above, given by the red solid lines. The theoretical predictions of the model given by Eq. (5) are shown by blue dashed lines. Laser-plasma parameters are given in the text.

cell sizes of $50 \times 80 \times 80 \mathrm{~nm}^{3}$. We use four particles per cell and have open boundaries throughout. After the magnetic field has been generated and before significant laser diffraction and filamentation occur, the moving window stops at $1 \mathrm{ps}$, and the evolution of the magnetic field is observed. Simulations were run for the $\left(\sigma_{z}=0, \ell=0\right),\left(\sigma_{z}=1, \ell=0\right)$, $\left(\sigma_{z}=0, \ell=1\right)$, and $\left(\sigma_{z}=0, \ell=2\right)$ laser modes, with parameters $P=65 \mathrm{TW}, \tau=100 \mathrm{fs}, \lambda=1 \mu \mathrm{m}, w_{0}=6 \mu \mathrm{m}$, and $n_{e}=3 \times 10^{19} \mathrm{~cm}^{-3}$. A fifth simulation with $\left(\sigma_{z}=0, \ell=1\right)$, $P=50 \mathrm{TW}, \tau=100 \mathrm{fs}, \lambda=1 \mu \mathrm{m}, w_{0}=7.2 \mu \mathrm{m}$, and $n_{e}=$ $3 \times 10^{19} \mathrm{~cm}^{-3}$ was also run to verify the decay model.

\section{A. Circularly polarized Gaussian simulations}

A simulation was first run for the linearly polarized Gaussian beam to verify the null magnetic field result and also to verify the absorption model. While we do not show the simulation results in this work, the null field was verified, and the absorption rate of this beam was found to be $0.43 \mathrm{~mm}^{-1}$, in excellent agreement with the predicted value of $0.435 \mathrm{~mm}^{-1}$ from Eq. (6). A second identical simulation was run but with a circularly polarized Gaussian beam $\left(\sigma_{z}=1, \ell=0\right)$ that gave a measured absorption slightly enhanced to $0.48 \mathrm{~mm}^{-1}$.

Given that the peak intensity of the $\mathrm{CP}$ Gaussian beam is $I_{0}=1.15 \times 10^{20} \mathrm{~W} \mathrm{~cm}^{-2}$, the interaction with the plasma is strongly nonlinear, with effects such as relativistic self-focusing, ponderomotive channeling, and filamentation strongly dominating the interaction. This can make it challenging to get clean magnetic field profiles out of the simulation, especially at later times, when the boundary conditions start to have an effect on the simulation. Because of this, we examine the magnetic field at early times before these nonlinear effects have time to sufficiently grow.

Figure 3 shows the axial magnetic field generation from the CP Gaussian mode at three early times in the simulation. Figures 3(a), 3(d), and 3(g) give the electron density normalized to the initial electron density at times of $0.6,0.77$, and 0.93 ps, respectively. Below these panels are transverse slices of the time averaged axial magnetic fields (averaged over 33 fs output dumps) relative to the laser pulse overlayed in green for each of the three times. The bottom three panels give radial lineouts of the time averaged magnetic field that have been averaged azimuthally, temporally (over $33 \mathrm{fs}$ ), and longitudinally between the red dashed lines in Figs. 3(b), 3(e), and 3(h). The simulation lineouts are given in red, whereas the theoretical prediction of Eq. (5) are given by the blue dashed line.

We find a remarkable overlap between the theory and the simulations at early times, but later, simulations diverge from the theory as the laser begins to diffract, self-focus, and filament. In particular we see the peak magnetic field strength become enhanced beyond the vacuum theory at $t=0.93 \mathrm{ps}$ due to the self-focusing. We also note the persistence of the 


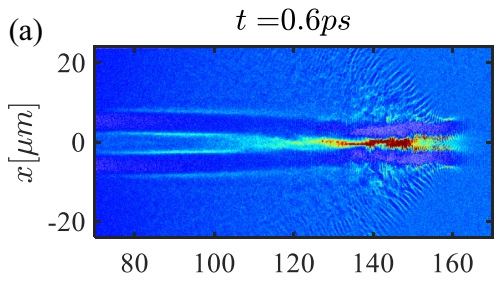

(b)
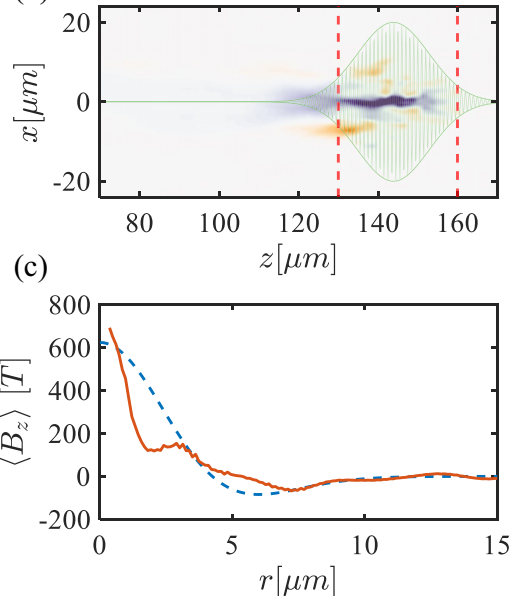

(d)

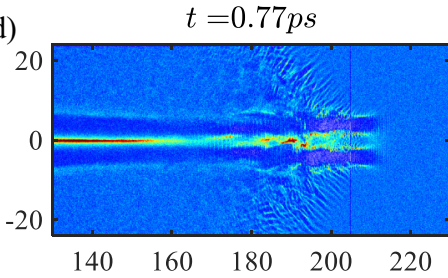

(e)

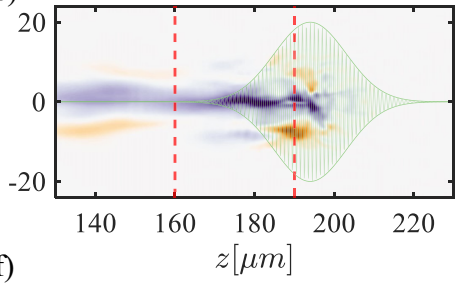

(f)

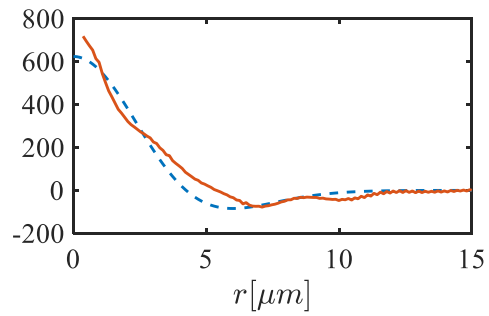

(g)

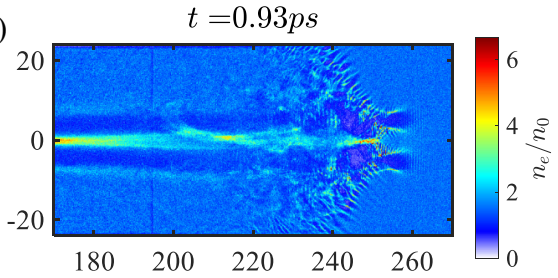

(h)
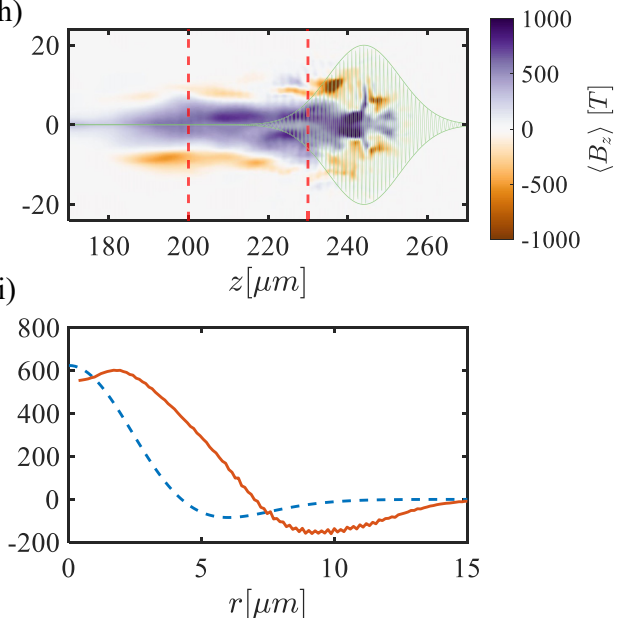

FIG. 4. Simulation results for LP $\ell=1$ Laguerre Gaussian driven magnetic fields at (from left to right) 0.6, 0.77, and 0.93 ps. (a), (d), and (g) Longitudinal electron density slice maps normalized to the initial electron density. (b), (e), and (h) Longitudinal axial magnetic field slice maps averaged over 33 fs; the laser pulse is overlayed in green, and lineout regions are given by the red dashed lines. (c), (f), and (i) Radial axial magnetic field lineouts averaged azimuthally, temporally, and longitudinally between the red dashed lines in the corresponding panel above, given by the red solid lines. The theoretical predictions of the model given by Eq. (5) are shown by blue dashed lines. Laser-plasma parameters are given in the text.

magnetic fields after the laser pulse has passed due to the residual magnetization of the plasma.

\section{B. Linearly polarized OAM simulations}

Next, simulations with LP OAM modes were run using both $\ell=1$ and $\ell=2$ modes, the results of which are given in Figs. 4 and 5, respectively, with the same layout described for the CP Gaussian beams in Fig. 3. The absorptions of the $\ell=1$ and $\ell=2$ modes were found to be 0.59 and $0.60 \mathrm{~mm}^{-1}$, respectively. This increase in absorption may come from the larger cross section of the OAM modes, from electron trapping within the donut mode leading to a more efficient laser-plasma energy transfer, or from a reduced ponderomotive channeling leading to a higher plasma density interacting and coupling with the laser directly.

Examining Fig. 4, we see a good overlap of the simulated magnetic field and that predicted by the theory. Like for the CP Gaussian simulations, we find the early times of the $\ell=1$ simulation agree well, but at later times as the laser starts to diffract, self-focus, and filament the agreement decreases. In Figs. 4(g) and 4(h), we see the magnetic field expand radially outwards without compromising its peak strength. This surprising result could be related to the compressed electron density on axis giving rise to a higher azimuthal current and a slow radial expansion outwards to fill the voids left by the ponderomotive force.
For the $\ell=2$ simulation shown in Fig. 5 we also see a good overlap between theory and simulation at early times, but at later times we find that the magnetic field amplifies to twice that of the predicted field while still maintaining its shape. This is likely due to the more complex self-focusing of the LG modes as the donut mode pinches into a tighter ring, both increasing intensity and maintaining the beam waist and then collapsing to a smaller beam waist at later times. During this self-focusing, additional angular momentum may be coupled to the plasma, giving rise to the enhanced magnetic field. As our model does not include self-focusing, we do not expect to reproduce the results at later times. There is some infilling of the null within the magnetic field on axis as the plasma thermalizes and then eventual radial expansion as the laser later diffracts.

In the current configuration the plasma is radially isotropic, and the axial magnetic field length is essentially limited to the Rayleigh length of the laser. For longer magnetic fields, one could use a preformed plasma channel to guide the laser and mitigate the effects of diffraction, but that is not explored in the current work.

Figures 6(a), 6(b), and 6(c) give the time averaged (averaged over 33 fs output dumps) axial magnetic field profile at times of $1,1.5$, and $2 \mathrm{ps}$, respectively, for the $\left(\sigma_{z}=0, \ell=1\right)$ mode. We find the magnetic field extends the length of the simulation box $(200 \mu \mathrm{m})$ to approximately equal to twice the Rayleigh range $\left(z_{0} \approx 113 \mu \mathrm{m}\right)$. 


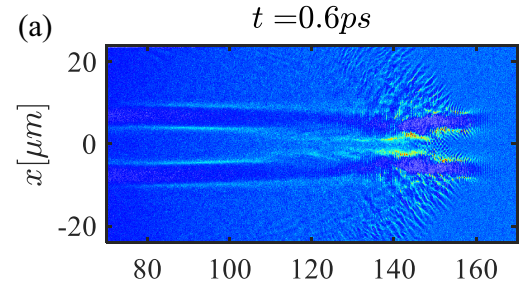

(b)
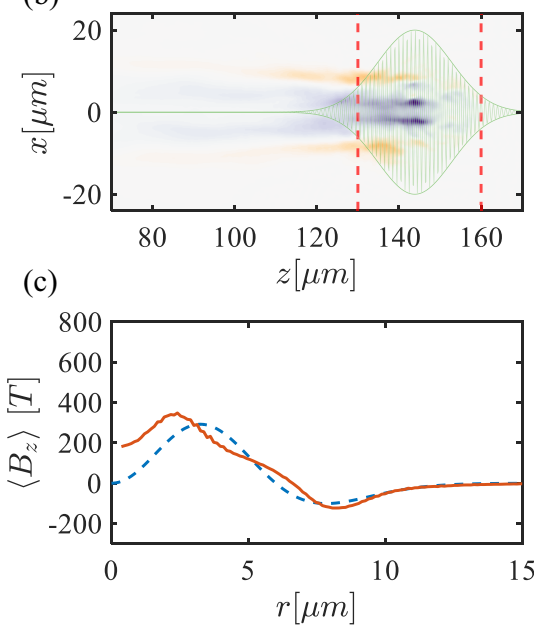

(d)

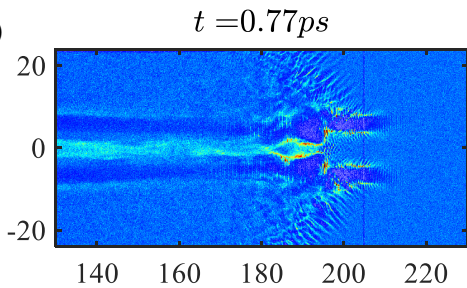

(e)

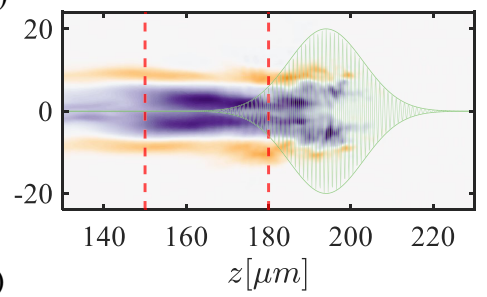

(f)

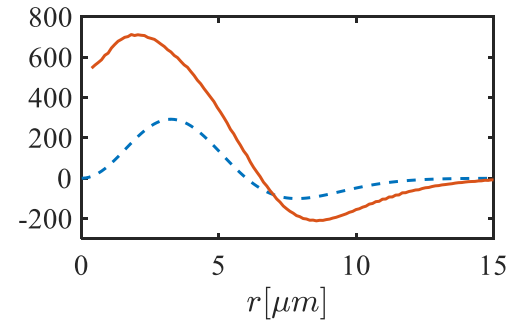

(g)

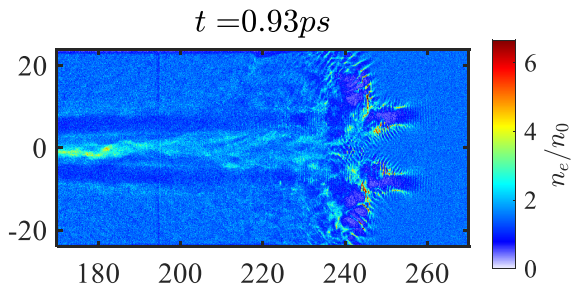

(h)

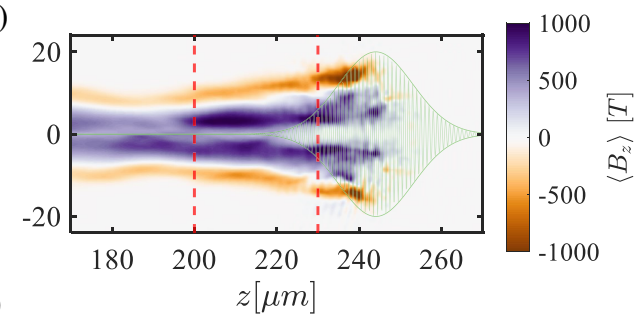

(i)

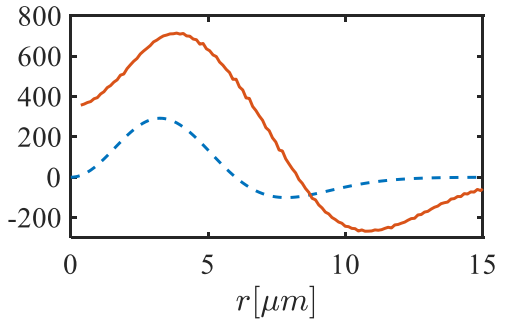

FIG. 5. Simulation results for LP $\ell=2$ Laguerre Gaussian driven magnetic fields at (from left to right) 0.6 , 0.77, and 0.93 ps. (a), (d), and (g) Longitudinal electron density slice maps normalized to the initial electron density. (b), (e), and (h) Longitudinal axial magnetic field slice maps averaged over $33 \mathrm{fs}$; the laser pulse is overlayed in green, and lineout regions are given by the red dashed lines. (c), (f), and (i) Radial axial magnetic field lineouts averaged azimuthally, temporally, and longitudinally between the red dashed lines in the corresponding panel above, given by the red solid lines. The theoretical predictions of the model given by Eq. (5) are shown by blue dashed lines. Laser-plasma parameters are given in the text.

At later times in Figs. 6(b) and 6(c), we find the $\ell=1$ mode begins to pinch, kink, and twist into a $3 \mathrm{D}$ springlike shape. Similar kinking is found for the $\left(\left|\sigma_{z}\right|=1,|\ell|=0\right)$ and $\left(\left|\sigma_{z}\right|=0,|\ell|=2\right)$ modes (not shown). Figures $6(\mathrm{~d}), 6(\mathrm{e})$, and 6 (f) show transverse slices of the electron azimuthal velocity time averaged over 33 fs. We see the azimuthal velocity map

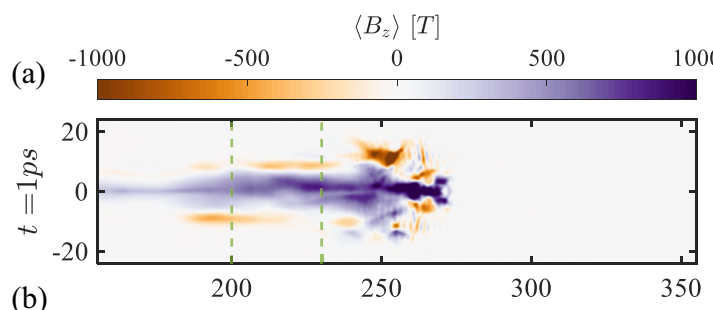

(b)
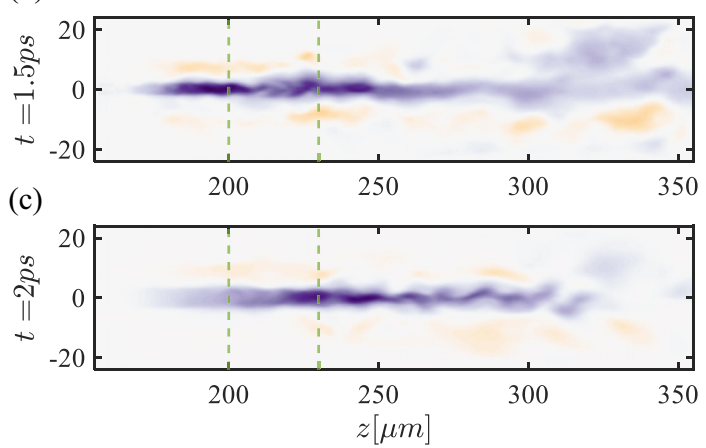

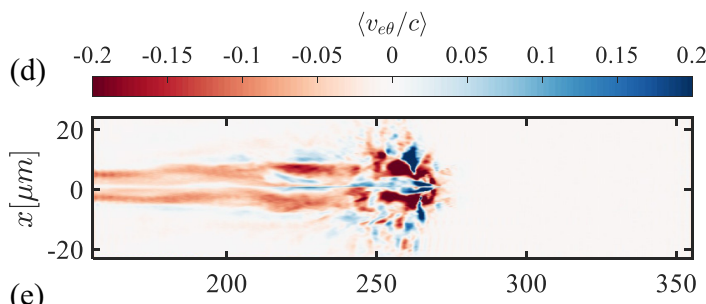

(e)
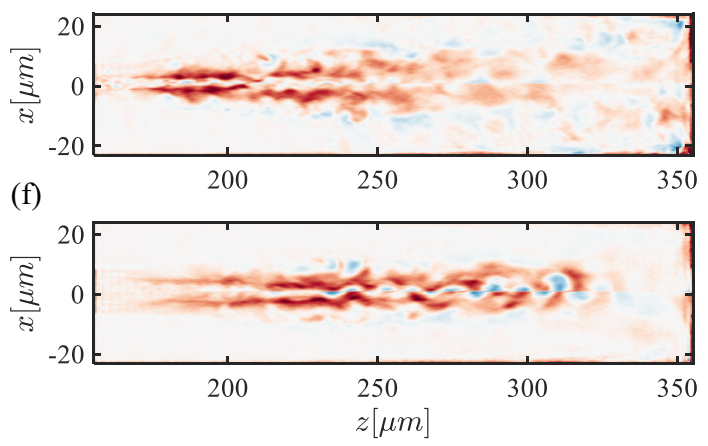

FIG. 6. Two-dimensional longitudinal slices of the time averaged (averaged over $33 \mathrm{fs}$ output dumps) axial magnetic field and the corresponding time averaged azimuthal electron velocity for the $\left(\sigma_{z}=0, \ell=1\right)$ OAM mode for times $t=1,1.5,2 \mathrm{ps}$. The green dashed lines indicate the regions used for sampling the field decay in Fig. 7. 
transition from a smooth cylindrical profile at 1 ps to a more turbulent and kinked map at 2 ps. This could be related to the wobble instability found in $\theta$-pinch configurations [29].

\section{MAGNETIC FIELD DECAY}

The lifetimes of the magnetic fields driven by the IFE at relativistic intensities in collisionless plasmas are poorly understood. If we consider the plasma beta $\beta=2 \mu_{0} n_{e} k_{B} T / B^{2}$, where $\mu_{0}$ is the permeability of free space, $k_{B}$ is Boltzmann's constant, and $T$ is the plasma temperature, we find that for magnetic field strengths of $1000 \mathrm{~T}$, electrons with energies $k_{B} T<100 \mathrm{keV}$ are trapped in the magnetic field, while higher energy electrons are able to escape, leading to the subsequent decay of the magnetic field.

To model the decay, we consider the motion of the hot electrons in helical motion with both azimuthal and radial velocity components. From Figs. 6(d)-6(f), we find the bulk of the hot electrons with OAM are born within a cylinder with a radius approximately equal to the peak intensity radius of the OAM mode $r_{\text {cyl }} \approx w_{0} \sqrt{\ell / 2}$. The higher order $|\ell| \geqslant 2$ modes are more complex with coaxial fields and will not be considered in our model.

The magnetic field as a function of time from a rotating cylinder of plasma can be derived from the Biot-Savart law [30]. The current density can be given as $\boldsymbol{j}=q n_{e}\left(v_{r} \hat{r}+v_{\theta} \hat{\theta}\right)$; the position vector can be given as $\boldsymbol{r}(t)=\left(v_{r} t+r_{0}\right) \hat{r}+z \hat{k}$, where $r_{0}$ is the initial electron radius, and $\hat{r}$ is the rotating polar coordinate. Inserting these into the Biot-Savart equation, we find

$$
B(t)=\frac{\mu_{0} e n_{e}}{2} \int_{0}^{c \tau} d z \int_{0}^{r_{\mathrm{cyl}}} \frac{v_{\theta}\left(v_{r} t+r_{0}\right) r_{0} d r_{0}}{\left[\left(v_{r} t+r_{0}\right)^{2}+z^{2}\right]^{3 / 2}} .
$$

We note that the axial length of the plasma for integration is restricted to approximately one pulse length $c \tau$. As the plasma is collisionless, we can assume the angular momentum acquired by the electrons is in the steady state; that is, $L_{z}=m_{e} v_{\theta} r_{0}$ is constant. Solving Eq. (8) is straightforward if we perform the radial integral first, yielding the result

$$
B(t)=B_{0}\left[\sinh ^{-1}\left(\frac{c \tau}{v_{r} t}\right)-\sinh ^{-1}\left(\frac{c \tau}{v_{r} t+r_{\mathrm{cyl}}}\right)\right] .
$$

Here, $B_{0}$ is the peak axial magnetic field at genesis.

To estimate the radial velocity of the electrons we can use the gradient of the plasma pressure [3]. To first order, the pressure can be attributed solely to the thermal pressure such that the force equation is given by

$$
\frac{n_{e} m_{i}}{Z} \frac{d v_{r}}{d t}=-\frac{\partial}{\partial r}\left(\frac{2}{3} \alpha_{\mathrm{abs}} \int I(t) d t\right) .
$$

Here, $m_{i}$ and $Z$ are the ion mass and ionization state, respectively. By using the ion mass, we assume the plasma is quasineutral, which was previously shown to be approximately valid on these timescales and for these laser intensities [3]. Integrating the laser temporal function is trivial, as shown earlier in the paper, leaving a second order ordinary differential equation (ODE),

$$
\frac{d^{2} r}{d t^{2}}+\frac{Z \alpha_{\mathrm{abs}} I_{0} \tau}{2 n_{e} m_{i}} \frac{\partial}{\partial r}\left|\psi_{\ell}\right|^{2}=0 .
$$

There are no known analytic solutions to Eq. (11), and we opt instead to use a parabolic approximation of $\psi_{\ell}$ given by

$$
\left|\psi_{\ell}\right|^{2} \approx A-\left(\frac{r}{w_{0}}-\sqrt{\frac{|\ell|}{2}}\right)^{2}
$$

where $A$ is an arbitrary amplitude scaling variable that is lost when we use the $\partial / \partial r$ operator.

The solution of the ODE is elementary using the parabolic approximation. Averaging the radial velocity over all positions within a beam waist, we find

$$
\left\langle v_{r}\right\rangle=\sqrt{\frac{Z \alpha_{\mathrm{abs}} I_{0} \tau}{4 \exp (2) n_{e} m_{i}}} .
$$

Substituting our previous simulation values, we find an average radial velocity of $\left\langle v_{r}\right\rangle \approx 0.01 c$. Given this, we can use the approximation that $(c \tau)^{2} \gg\left(v_{r} t\right)^{2}$ given the timescale of the magnetic field decay is observed to be on the order of a picosecond, allowing us to simplify Eq. (9) to

$$
\frac{B\left(t-t_{0}\right)}{B_{0}} \approx \ln \left(1+\frac{1}{t / t_{D}+0.582}\right) .
$$

Here, $t_{D}=w_{0} /\left(v_{e r} \sqrt{2}\right)$ is the decay parameter for the $|\ell|=1$ mode, and $t_{0}$ is the genesis time. The factor of 0.582 is set such that $B(0)=B_{0}$. Combining the results of Eqs. (13) and (14) allows us to derive the scaling law for the time decay parameter of the $|\ell|=1$ driven magnetic fields,

$$
t_{D}[\mathrm{ps}] \approx \frac{1}{8} \frac{w_{0}^{2}[\mu \mathrm{m}]}{\lambda[\mu \mathrm{m}]} \sqrt{\frac{A}{Z}} \frac{1}{\sqrt{P[\mathrm{TW}]}},
$$

where $A$ is the atomic mass number of the plasma. While this model is not valid for $|\ell| \geqslant 2$, it could be suitable for also modeling the $\mathrm{CP} \ell=0$ mode given its similarities to the $|\ell|=$ 1 magnetic field profile.

Using our simulation values, we estimate the decay parameter as $t_{D}=0.8 \mathrm{ps}$. Determining the decay time numerically is challenging due to the magnetic field instabilities, as well as laser self-focusing causing the magnetic field to be dynamic on axis. We therefore radially, azimuthally, and longitudinally average the magnetic field through a cylinder of radius $r_{\text {cyl }}=$ $w_{0} / \sqrt{2}$ and length $c \tau$. The longitudinal region is indicated by the dashed green lines from $z=200$ to $230 \mu \mathrm{m}$ shown in Fig. 6. The average value of the magnetic field in this region is plotted as a function of time in Fig. 7 for three simulations. The simulation for the $65 \mathrm{TW},\left(\sigma_{z}=0, \ell=1\right), w_{0}=6 \mu \mathrm{m}$ mode is shown with blue circles, whereas the $50 \mathrm{TW},\left(\sigma_{z}=\right.$ $0, \ell=1)$, and $w_{0}=7.2 \mu \mathrm{m}$ simulation is shown with red diamonds. The purple crosses represent the data from the initial CP Gaussian $\left(\sigma_{z}=1, \ell=0\right)$ simulation with $P=65 \mathrm{TW}$, $w_{0}=6 \mu \mathrm{m}$.

Using a nonlinear least squares fit of Eq. (14) to the data, we find a numerical decay parameter of $0.86 \mathrm{ps}$ for the $\sigma_{z}=$ $0, \ell=1, w_{0}=6 \mu \mathrm{m}$ mode, shown by the red dashed line in Fig. 7. This is in very good agreement with the analytic estimate of $0.8 \mathrm{ps}$. Considering the $\sigma_{z}=0, \ell=1, w_{0}=7.2 \mu \mathrm{m}$ case with a laser power of $50 \mathrm{TW}$, we predict a decay parameter of $\tau=1.3 \mathrm{ps}$ and numerically find a value of $1.18 \mathrm{ps}$, shown by the green dashed line in Fig. 7 . 


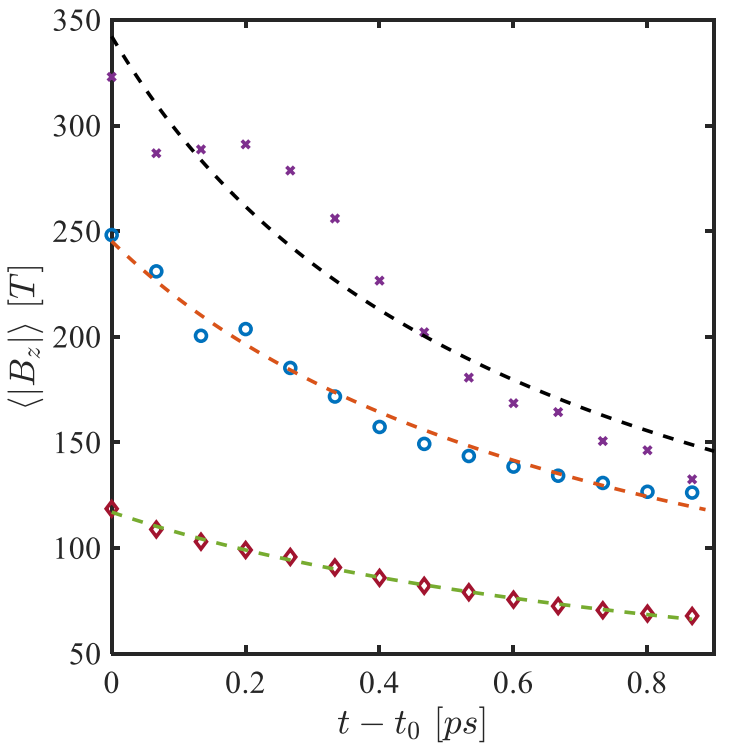

FIG. 7. Average magnetic field within a cylinder of radius $r_{\mathrm{cyl}}=$ $w_{0} / \sqrt{2}$ and length $30 \mu \mathrm{m}$ from $z-200-230 \mu \mathrm{m}$ as a function of time. The LP $65 \mathrm{TW}, \ell=1, w_{0}=6 \mu$ m mode is given by the blue circles and fitted with the red dashed line with decay parameter $t_{D}=0.86 \mathrm{ps}$. The LP $50 \mathrm{TW}, \ell=1, w_{0}=7.2 \mu \mathrm{m}$ mode is given by the red diamonds and fitted with the green dashed line with decay parameter $t_{D}=1.18 \mathrm{ps}$. The $65 \mathrm{TW}, \sigma_{z}=1, \ell=0, w_{0}=6 \mu \mathrm{m}$ mode is given by the purple crosses and fitted with the black dashed line with decay parameter $t_{D}=0.69 \mathrm{ps}$.

Finally, for the $\left(\sigma_{z}=1, \ell=0\right)$ case we find a predicted decay of $0.8 \mathrm{ps}$ and numerically find a decay time of $0.69 \mathrm{ps}$, as shown by the black dashed line. This reduced decay time could be due to the stronger self-focusing of the CP Gaussian mode as it has a higher peak intensity than the LG modes. The increased self-focusing results in a smaller beam waist and hence a shorter decay time.

Given the dependence of the decay time on the laser beam waist, one could control the magnetic field lifetime by adjusting the $f$ number to higher values at a cost of magnetic field strength. An optimal $f$ number is therefore needed to balance the magnetic field strength, axial length, and decay time to suit a given application of interest. Alternatively, one could adjust the decay time of the magnetic field by changing the plasma to a heavier ion species.

\section{SUMMARY}

In summary, we have successfully demonstrated and characterized the IFE with circularly polarized Gaussian and linearly polarized OAM modes over large spatial and temporal scales. We have derived a description of OAM driven magnetic fields in underdense plasma and introduced a model for the subsequent decay of the magnetic field. Magnetic fields with strengths up to $1 \mathrm{kT}$ extending up to $200 \mu \mathrm{m}$ and persisting for several picoseconds have been demonstrated. Simulations indicated an increased absorption rate when using increased OAM mode numbers. The decay time, axial length, and magnetic field strength are all functions of the laser beam waist and can be optimized to suit an experiment as such. Plasma channel guiding of the laser pulse could extend the magnetic field over several Rayleigh lengths and would instead be limited to the pump depletion length.

With the recent demonstration of high intensity OAM modes in high power laser facilities with parameters similar to those assumed in this work, experimental verification of the magnetic fields could now be feasible [16]. Generation and control of multi-kilo-Tesla magnetic fields will open up new opportunities in a number of areas of high energy density physics and laboratory astrophysics, including particle acceleration schemes, magnetic reconnection, fast ignition, and fundamental physics.

\section{ACKNOWLEDGMENTS}

This work was performed under the auspices of the U.S. Department of Energy by Lawrence Livermore National Laboratory under Contract No. DE-AC52-07NA27344, supported by the Natural Sciences and Engineering Research Council of Canada (Grant No. RGPIN-2019-05013), and support provided by WestGrid and Compute Canada. This work was in part funded by UK EPSRC Grants No. EP/G054950/1, No. EP/G056803/1, No. EP/G055165/1, and No. EP/M022463/1. The authors would like to thank and acknowledge J. Myatt, J. Ludwig, and P. Michel for helpful discussions.
[1] Z. Najmudin, M. Tatarakis, A. Pukhov, E. L. Clark, R. J. Clarke, A. E. Dangor, J. Faure, V. Malka, D. Neely, M. I. K. Santala, and K. Krushelnick, Measurements of the Inverse Faraday Effect from Relativistic Laser Interactions with an Underdense Plasma, Phys. Rev. Lett. 87, 215004 (2001).

[2] Z. Lécz, A. Andreev, and A. Seryi, Plasma rotation with circularly polarized laser pulse, Laser Part. Beams 34, 31 (2016).

[3] M. G. Haines, Generation of an Axial Magnetic Field from Photon Spin, Phys. Rev. Lett. 87, 135005 (2001).

[4] N. Naseri, V. Y. Bychenkov, and W. Rozmus, Axial magnetic field generation by intense circularly polarized laser pulses in underdense plasmas, Phys. Plasmas 17, 083109 (2010).
[5] A. A. Frolov, Excitation of magnetic fields by a circularly polarized laser pulse in a plasma channel, Plasma Phys. Rep. 30, 698 (2004)

[6] V. Y. Bychenkov and V. Tikhonchuk, Magnetic field generation by short ultraintense laser pulse in underdense plasmas, Laser Part. Beams 14, 55 (1996).

[7] Z. M. Sheng and J. Meyer-ter-Vehn, Inverse Faraday effect and propagation of circularly polarized intense laser beams in plasmas, Phys. Rev. E 54, 1833 (1996).

[8] Z. Lecz, I. Konoplev, A. Seryi, and A. Andreev, Gigagauss solenoidal magnetic field inside bubbles excited in under-dense plasma, Sci. Rep. 6, 36139 (2016).

[9] J. Deschamps, M. Fitaire, and M. Lagoutte, Inverse Faraday Effect in a Plasma, Phys. Rev. Lett. 25, 1330 (1970). 
[10] Y. Horovitz, S. Eliezer, A. Ludmirsky, Z. Henis, E. Moshe, R. Shpitalnik, and B. Arad, Measurements of Inverse Faraday Effect and Absorption of Circularly Polarized Laser Light in Plasmas, Phys. Rev. Lett. 78, 1707 (1997).

[11] S. Ali, J. R. Davies, and J. T. Mendonca, Inverse Faraday Effect with Linearly Polarized Laser Pulses, Phys. Rev. Lett. 105, 035001 (2010).

[12] R. Nuter, P. Korneev, I. Thiele, and V. Tikhonchuk, Plasma solenoid driven by a laser beam carrying an orbital angular momentum, Phys. Rev. E 98, 033211 (2018).

[13] R. Nuter, P. Korneev, E. Dmitriev, I. Thiele, and V. T. Tikhonchuk, Gain of electron orbital angular momentum in a direct laser acceleration process, Phys. Rev. E 101, 053202 (2020).

[14] L. Allen, M. W. Beijersbergen, R. J. C. Spreeuw, and J. P. Woerdman, Orbital angular momentum of light and the transformation of Laguerre-Gaussian laser modes, Phys. Rev. A 45, 8185 (1992).

[15] V. Tikhonchuk, P. Korneev, E. Dmitriev, and R. Nuter, Numerical study of momentum and energy transfer in the interaction of a laser pulse carrying orbital angular momentum with electrons, High Energy Density Phys. 37, 100863 (2020).

[16] A. Longman, C. Salgado, G. Zeraouli, J. I. Apinaniz, J. A. Pérez-Hernández, M. K. Eltahlawy, L. Volpe, and R. Fedosejevs, Off-axis spiral phase mirrors for generating high-intensity optical vortices, Opt. Lett. 45, 2187 (2020).

[17] D. Wu and J. W. Wang, Magnetostatic amplifier with tunable maximum by twisted-light plasma interactions, Plasma Phys. Controlled Fusion 59, 095010 (2017).

[18] Y. Shi, J. Vieira, R. M. G. M. Trines, R. Bingham, B. F. Shen, and R. J. Kingham, Magnetic Field Generation in Plasma Waves Driven by Copropagating Intense Twisted Lasers, Phys. Rev. Lett. 121, 145002 (2018).

[19] A. Longman and R. Fedosejevs, Optimal Laguerre-Gaussian modes for high-intensity optical vortices, J. Opt. Soc. Am. A 37, 841 (2020).
[20] S. M. Barnett and L. Allen, Orbital angular momentum and nonparaxial light beams, Opt. Commun. 110, 670 (1994).

[21] A. Longman and R. Fedosejevs, Mode conversion efficiency to Laguerre-Gaussian OAM modes using spiral phase optics, Opt. Express 25, 17382 (2017).

[22] F. Mackenroth, A. R. Holkundkar, and H.-P. Schlenvoigt, Ultra-intense laser pulse characterization using ponderomotive electron scattering, New J. Phys. 21, 123028 (2019).

[23] H. Akou and A. S. Firouzjaei, Direct electron bunch acceleration by Laguerre-Gauss laser pulse, Phys. Plasmas 27, 093102 (2020).

[24] Y. Shi, D. Blackman, D. Stutman, and A. Arefiev, Generation of Ultrarelativistic Monoenergetic Electron Bunches via a Synergistic Interaction of Longitudinal Electric and Magnetic Fields of a Twisted Laser, Phys. Rev. Lett. 126, 234801 (2021).

[25] S. Miyazaki, S. Kawata, Q. Kong, K. Miyauchi, K. Sakai, S. Hasumi, R. Sonobe, and T. Kikuchi, Generation of a microelectron beam by an intense short pulse laser in the Tem(1,0)Tem(0,1) mode in vacuum, J. Phys. D 38, 1665 (2005).

[26] W. Lu, M. Tzoufras, C. Joshi, F. S. Tsung, W. B. Mori, J. Vieira, R. A. Fonseca, and L. O. Silva, Generating multi-GeV electron bunches using single stage laser Wakefield acceleration in a 3D nonlinear regime, Phys. Rev. ST Accel. Beams 10, 061301 (2007).

[27] C. D. Decker, W. B. Mori, K. Tzeng, and T. Katsouleas, The evolution of ultra-intense, short-pulse lasers in underdense plasmas, Phys. Plasmas 3, 2047 (1996).

[28] T. D. Arber, K. Bennett, C. S. Brady, A. Lawrence-Douglas, M. G. Ramsay, N. J. Sircombe, P. Gillies, R. G. Evans, H. Schmitz, A. R. Bell, and C. P. Ridgers, Contemporary particle-in-cell approach to laser-plasma modelling, Plasma Phys. Controlled Fusion 57, 113001 (2015).

[29] J. P. Freidberg and L. D. Pearlstein, Rotational instabilities in a theta pinch, Phys. Fluids 21, 1207 (1978).

[30] J. D. Jackson, Classical Electrodynamics, 3rd ed. (Wiley, New York, 1999). 